\author{
Nubia Esperanza Hernández Bernal 1,* \\ Adriana Patricia Bulla² \\ Eliana Mancilla López ${ }^{3}$ \\ Laura Vanessa Peña Peña4
}

1. Enfermera. Magíster en Enfermería. Docente de la Escuela de Enfermería de la Universidad Pedagógica y Tecnológica de Colombia. Tunja. Colombia.

2. Enfermera. Especialista en seguridad y salud en el trabajo. Docente de la Escuela de Enfermería de la Universidad Pedagógica y Tecnológica de Colombia. Tunja. Colombia.

3. Enfermera. Unidad Renal. San Gil. Colombia.

4. Enfermera. Candidata a Magíster en Salud Pública. Asistente de Investigación. Bogotá. Colombia.

*Autor para correspondencia.

Correo electrónico: nubia.hernandez01@uptc.edu.co (Nubia Esperanza Hernández Bernal).

Recibido el 21 de octubre de 2019; aceptado el 9 de diciembre de 2019.

\section{Prevalencia de lesiones de piel asociadas a la dependencia física en geriátricos e instituciones de salud de Tunja}

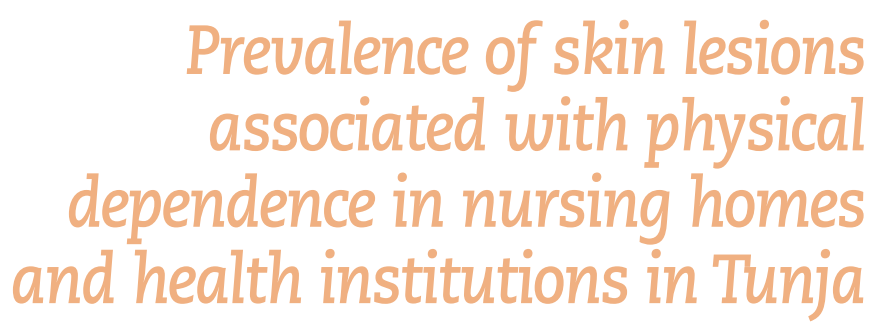

\section{RESUMEN}

Objetivos: Determinar la prevalencia puntual de lesiones de piel asociadas a la dependencia física, realizar caracterización sociodemográfica de las personas afectadas, caracterizar las lesiones con base en disposiciones del Grupo Nacional para el Estudio y Asesoramiento en Úlceras por Presión y Heridas Crónicas

(GNEAUPP), el National Pressure Ulcer Advisory Panel (NPUAP) y el European Pressure Ulcer Advisory Panel (EPUAP) y establecer la habilidad del personal de salud para el reconocimiento y clasificación de las lesiones de piel asociadas a dependencia. Metodología: Estudio multicéntrico descriptivo de prevalencia puntual en instituciones de servicios de salud de tercer nivel y centros gerontológicos de Tunja, Colombia. Resultados: Participaron cuatro instituciones. La muestra fue de 409 personas, 24 de las cuales presentaron lesiones de piel asociadas a la dependencia para una prevalencia del 5,86\%. De los afectados, el 41,6\% eran hombres y el 58,4\% mujeres; el 79,1\% con 50 años o más; en cuanto a la procedencia, el 53,8\% pertenecía al área urbana y el $46,2 \%$ a la rural; un $56,6 \%$ de los pacientes pertenecían al régimen subsidiado, el $30 \%$ al contributivo y $13,4 \%$ al especial. La etiología de las lesiones estuvo discriminada de la siguiente manera: por presión, 67,3\%; LESCAH, 13,4\%; MARSI, 11,5\% y desgarros cutáneos, 7,7\%. Conclusiones: la prevalencia encontrada se relaciona con resultados de estudios colombianos previos, la afectación es mayor en mujeres y la mayor frecuencia de ocurrencia de lesiones corresponde a las ocasionadas por la presión y cizalla; las lesiones de piel asociadas a la dependencia se presentaron con mayor frecuencia en personas de procedencia urbana. El $75 \%$ de las personas que diligenciaron la encuesta reportaron no haber recibido capacitación institucional para identificación y clasificación de lesiones de piel asociadas a la dependencia.

PALABRAS CLAVE: Prevalencia, estudio de prevalencia, piel, heridas y lesiones, atención de enfermería, investigación en enfermería clínica.

\section{ABSTRACT}

Objectives: To determine the exact prevalence of skin lesions associated with dependence physical, conduct socio-demographic characterization of those affected, characterizing lesions based on provisions of the National Group for the Study and advice on Pressure Ulcers and Chronic Wounds (GNEAUPP), the National Pressure Ulcer Advisory Panel (NPUAP) and the European Pressure Ulcer Advisory Panel (EPUAP), establish the ability of health personnel to recognize and classify lesions associated with dependence.

Methodology: descriptive multicenter point prevalence in third level health care institutions and gerontology centers from Tunja, Colombia. Results: four institutions participated, the sample was 409 people, 24 had lesions associated with dependence for a prevalence of $5.86 \%$; of those affected $41.6 \%$ were men and $58.4 \%$ women, $79.1 \%$ with $\geq 50$ years regarding the origin $53.8 \%$ belonged to urban zones and $46.2 \%$ rural zones; $56.6 \%$ of patients belonged to the subsidized health scheme, $30 \%$ to contributory health scheme $13.4 \%$ to special. The etiology of lesions was discriminated as follows: pressure $67.3 \%$; LESCAH 13.4\%; MARSI 11.5\% and 7.7\% skin tears. Conclusions: The prevalence found is related to results of Colombian previous studies, the effect is greater in women and increased frequency of occurrence of injuries corresponds to those caused by pressure and mechanical friction, LPAD occurred more often in people of origin urban. $75 \%$ of people who completed the survey reported not having received institutional training for identification and classification (LPAD).

KEYWORDS: Prevalence, prevalence study, skin, wounds and injuries, nursing care, clinical nursing research.

\section{У INTRODUCCIÓN}

Las lesiones cutáneas asociadas a la dependencia (LPAD) se generan como resultado de la condición propia del ser humano, cuando este pierde de forma permanente o transitoria su autonomía y capacidad para realizar acciones de autocuidado, así como las actividades básicas de la vida diaria. Se presentan en pacientes críticos o crónicos en postración, en los cuales actúan agentes etiológicos inherentes a la persona, al cuidado de la piel, así como al uso de dispositivos médico-sanitarios fundamentales para el manejo de la situación de salud ${ }^{1,2}$. 
A nivel internacional, existen diferentes organismos, como el estadounidense NPUAP (National Pressure Ulcer Advisory Panel) y el europeo EPUAP (European Pressure Ulcer Advisory Panel) que han generado documentos y lineamientos argumentados de la conceptualización, prevención, clasificación y manejo de lesiones por presión (LPP); sin embargo, en 2014, García Fernández et al. propusieron un modelo teórico en el que sugerían la existencia de diferentes tipos de lesiones, explicaban su mecanismo de producción y desvirtuaban la presión como único agente causal; así mismo, afirmaban que la dependencia física es el común denominador de estas lesiones en la piel. Al referirse a la multicausalidad incluían, además de la presión, la cizalla y su combinación, la humedad, el roce y la fricción ${ }^{1}$.

El Grupo Nacional para el Estudio y Asesoramiento de las Úlceras por Presión y Heridas Crónicas (GNEAUPP), que constituye una autoridad internacional en el tema, adoptó la propuesta y formuló a la comunidad científica un documento técnico de consenso de clasificación-categorización de las lesiones relacionadas con la dependencia que incluye: las lesiones por presión (LPP) y cizalla, lesiones cutáneas asociadas a la humedad (LESCAH), lesiones por roce/fricción y las lesiones mixtas o combinadas ${ }^{2}$.

El GNEAUPP define las úlceras por presión y cizalla como "una lesión localizada en la piel y/o tejido subyacente por lo general sobre una prominencia ósea, como resultado de la presión, o la presión en combinación con las fuerzas de cizalla. En ocasiones también pueden aparecer sobre tejidos blandos sometidos a presión externa por diferentes materiales o dispositivos clínicos" ${ }^{2}$, los cuales se utilizan para fines diagnósticos y terapéuticos, como sondas, cánulas y catéteres vesicales. Las lesiones conocidas como MARSI (Medical Adhesive Related Skin Injuries), lesiones cutáneas relacionadas con adhesivos médicos, se producen por la fuerza de tensión con que se sitúa el dispositivo, las arrugas en la piel debajo de este, la técnica al retirarlo o la reacción propia del epitelio a los componentes del producto ${ }^{3}$. En las lesiones identificadas como LESCAH, los agentes causales son: exceso de humedad, irritación física e irritación química sobre la piel ${ }^{4}$. El GNEAUPP las define como "la inflamación y/o erosión de la piel causada por la exposición prolongada/excesiva a la humedad, incluyendo orina, heces líquidas o exudado de las heridas”. El mismo grupo define las lesiones por roce o fricción como "la lesión localizada en la piel provocada por las fuerzas derivadas del roce-fricción entre la piel del paciente y otra superficie paralela que, en contacto con él, se mueven ambas en sentido contrario" 2 .

Las lesiones descritas se consideran una problemática subregistrada, permanente y silenciosa de salud pública, que genera afectaciones para las personas en sus dimensiones fisiológica, psíquica, familiar y social, ya que suponen un franco deterioro de la calidad de vida. La persona y su familia asumen elevados gastos económicos y humanos, relacionados con tratamientos, insumos, desplazamientos, estancias hospitalarias ${ }^{5}$, así como procesos prolongados de recuperación y rehabilitación; en cuanto a la dimensión social, se limita la adopción de roles y la reintegración social, por complicaciones que implican reingresos hospitalarios y manejos extensos y dolorosos ${ }^{6-8}$.

Adicionalmente, repercuten en las dinámicas institucionales, dado que conllevan el incremento en el uso de insumos médico-quirúrgicos, recursos estructurales, sobrecarga laboral para el personal sanitario, elevados costos económicos para los sistemas de salud por la disminución en el giro cama y el redireccionamiento de recursos para su abordaje $e^{8,9}$. Ante esta situación, las autoridades sanitarias colombianas acogidas a los modelos internacionales vienen proyectando lineamientos y estrategias encaminadas a reducir dichos impactos y a garantizar la seguridad del paciente $^{10}$.
En cuanto al comportamiento de la prevalencia de las lesiones cutáneas a nivel internacional se encuentra pluralidad de reportes. Así, en un hospital de Noruega se realizó la búsqueda exclusiva de LPP, encontrando una prevalencia de periodo del 14,9\%, con mayor frecuencia de afectación en el sacro y en el talón ${ }^{11}$. En Estados Unidos y Canadá, un estudio internacional de prevalencia de LPP asociadas a dispositivos médicos evidenció una prevalencia del 9,9\% de pacientes que presentaron LPP. Las unidades donde hubo mayor prevalencia fueron: hospitalización, con un 0,60\%, y la unidad de cuidados intensivos y rehabilitación con un $0,59 \%{ }^{12}$. En los mismos países, la prevalencia por dermatitis asociada a la incontinencia (DAI), que hace parte de las LESCAH, fue del $4,3 \%{ }^{13}$. Un estudio reveló una prevalencia del 10,8\% en LPP específicamente para Australia ${ }^{14}$. Así mismo, en países como Reino Unido, Bélgica, Suecia y Australia, la prevalencia de UPP adquiridas a nivel hospitalario oscila alrededor del 15\%; sin embargo, este dato podría variar con base a la definición de UPP y la metodología de la detección ${ }^{15}$.

En el V Estudio Nacional de Prevalencia de Lesiones por Presión y otras lesiones cutáneas relacionadas con la dependencia, realizado en Espańa en 2017, el cual se puede considerar referente internacional, se reportó una prevalencia global del 8,7\%; se destaca que, en el marco de los estudios espańoles realizados, es la primera oportunidad en que se incluyen todas las lesiones asociadas con la dependencia propuestas en el modelo de García Fernandez ${ }^{1}$. En este sentido, la prevalencia encontrada según el tipo de lesión fue del 7\% para LPP, del 1,4\% para LESCAH, del $0,9 \%$ para lesiones por fricción y del $1,5 \%$ para lesiones combinadas, entre otras. En general se presenta un discreto predominio de prevalencia aumentada en hombres; la edad promedio de afectación fue 74,7 años. Respecto a la zona anatómica, el lugar de mayor prevalencia en todas las lesiones cutáneas analizadas fue el sacro-coxis, seguido de los trocánteres e isquiones; según la categorización, las más frecuentes fueron: en las LPP, las de grado II; en LESCAH, las IA; en las relacionadas con la fricción, la categoría I; y en cuanto a las combinadas, la categoría más frecuente fue la categoría $2^{16}$.

En la ciudad de Burgos (España), en una investigación que incluyó alteraciones cutáneas por humedad y por presión, se encontró una prevalencia del 5,69\% para LESCAH y del 21,53\% para LPP, de las cuales el $93,56 \%$ estaban en estadios I y II ${ }^{17}$. En Navarra (España), se determinó la prevalencia puntual de LPP en una clínica donde se valoraron 138 pacientes del 87,3\% de los pacientes ingresados, y se determinó una prevalencia del 8,8\% ${ }^{18}$. En Valencia (España) la prevalencia de LPP fue del 3,4\%, con predilección por zonas anatómicas como talón y sacro; en cuanto a la gravedad, prevaleció el estadio III en el $50 \%$ de los pacientes afectados $^{19}$.

En México, en un hospital de tercer nivel, la prevalencia de LPP fue del $13,81 \%$, coincidiendo con mayor afectación en la zona del talón y sacro, principalmente en personas con edad igual o superior a 65 ańos ${ }^{20}$, dato epidemiológico que difiere de lo encontrado en un hospital de segundo nivel en el mismo país con una prevalencia bruta de LPP del $28 \%$ y una incidencia del $13 \%{ }^{8}$. En un municipio brasileño se reportó una prevalencia de LPP del 5\%, y la edad promedio de las personas afectadas fue de 71,1 años ${ }^{21}$. Así mismo, en una revisión sistemática se reporta un estudio que revela una prevalencia de UPP del 35\% en Belo Horizonte, Brasil ${ }^{22}$.

Una investigación colombiana incluyó once departamentos y reportó una prevalencia total de lesiones de piel del 5,2\%, discriminada de la siguiente manera: $43,1 \%$ para LPP, $10,2 \%$ para DAI, $10,8 \%$ para MARSI, 5,5\% para desgarros cutáneos, 11,4 para lesiones por pie diabético, $14,6 \%$ úlceras en pierna de origen venoso y $4,4 \%$ úlcera en pierna de origen arterial ${ }^{23}$. En otro estudio colombiano, donde participaron 
veintidós departamentos, se encontró una prevalencia de periodo del $2,2 \%$, con un considerable aumento de afectación en hombres, con el $67 \%$ frente al $33 \%$ presentado en mujeres. En cuanto al agente causal, la presión afecta al $98 \%$ de los casos, la cizalla al $37 \%$, la humedad al $26 \%$ y la incontinencia al $24 \%$, aunque en algunos casos se reportó una combinación de varias causas ${ }^{24}$. En Bogotá se halló una prevalencia del $14,1 \%$, con predominio de afectación en el género masculino, y la causa más frecuente fue la presión, con un 92,5\%, seguido de las lesiones ocasionadas por la humedad ${ }^{25}$.

Hay que puntualizar que en los estudios nacionales no está incluido el departamento de Boyacá o la ciudad de Tunja. Igualmente, en la literatura científica no existen reportes de la problemática para el contexto, por lo que se desconocen indicadores epidemiológicos a nivel local en cuanto a pacientes afectados y las características de las lesiones.

En los últimos años, en Colombia se han trabajado políticas públicas para garantizar la seguridad del paciente; de esta manera, el Ministerio de Salud y Protección Social propone la guía técnica de buenas prácticas para la seguridad del paciente en la atención en salud, en la que se aborda la prevención de las LPP como un proceso asistencial seguro ${ }^{10}$. Sin embargo, la ausencia de indicadores epidemiológicos locales afecta a la generación de estrategias para la prevención y abordaje de la situación y, en consecuencia, producción de resultados efectivos.

Por tal motivo, urge la vigilancia epidemiológica de las LPAD, dado que estas requieren un profundo análisis que derive en planes de mejoramiento para consolidar la calidad en la atención y el cuidado de las personas en los diferentes escenarios clínicos y ambulatorios; lo anterior concuerda con las políticas de seguridad del paciente que se soportan en revisiones de este aspecto, como el estudio de prevalencia de efectos adversos en hospitales de Latinoamérica IBEAS, donde se consideran las LPAD como evento adverso en salud, concebido como altamente prevenible $e^{26}$.

Para el profesional de enfermería que lidera, ejecuta y gestiona el cuidado de la persona 24 horas al día, es fundamental adjudicarse y apropiarse del rol de educador en salud, con énfasis en la prevención y la implementación de métodos de cuidado seguros individualizados e integrales, que generen indicadores epidemiológicos de las lesiones cutáneas, fiables y completos; además, es indispensable reestructurar la visión del enfermero(a) incluso desde la academia donde se proyecten para el cuidado en la cabecera de la cama del paciente con contribución dinámica en la planeación, ejecución, direccionamiento y evaluación de todas las intervenciones de cuidado.

\section{Y OBJETIVOS}

\section{Objetivo principal}

- Determinar la prevalencia puntual de lesiones de piel asociadas a dependencia física en instituciones de salud y geriátricos de Tunja.

\section{Objetivos secundarios}

- Realizar la caracterización sociodemográfica de las personas con lesiones de piel asociadas a la dependencia física.

- Caracterizar las lesiones que afectan a las personas con dependencia física en Tunja, con base en lo dispuesto por el GNEAUPP, el NPUAP y el EPUAP.

- Determinar la habilidad por parte del personal de salud encuestado para el reconocimiento y clasificación de las lesiones de piel asociadas a dependencia.

\section{METODOLOGÍA}

Se realizó un estudio multicéntrico descriptivo de prevalencia de punto, el cual se llevó a cabo en instituciones con servicio de hospitalización. Así: clínica de carácter privado y hospital público correspondientes al tercer nivel de atención (según la estructura del sistema de salud de la República de Colombia) y dos centros gerontológicos de la ciudad de Tunja, con la aprobación del comité de ética o de las directivas de las entidades para el uso de la información.

Se diseñó una encuesta para la recolección de datos que se aplicó a personal profesional y técnico de enfermería encargado del cuidado de la piel de las personas hospitalizadas en las instituciones participantes, así como al personal asignado al manejo de la información institucional y de las personas afectadas por LPAD; posteriormente se realizó la inspección de la piel y de las heridas así como la verificación de datos en las historias clínicas por parte de las enfermeras o técnicos de enfermería que atendieron la encuesta, quienes eran responsables del cuidado de cada uno de los pacientes afectados. Con el fin de orientar la identificación y clasificación de las lesiones, se elaboró una guía con fotografías que ilustraban los diferentes tipos de LPAD y sus categorías.

La encuesta se distribuyó en secciones de la siguiente manera: sección I, incluyó ocho preguntas relativas a la institución, como carácter (público o privado), nivel de atención, número de pacientes hospitalizados, índice ocupacional y responsable del programa o del cuidado de los pacientes; la sección II comprendió 6 preguntas concernientes a método y escala utilizada para la medición del riesgo de desarrollar LPAD, conocimiento para la clasificación de lesiones asociadas a la dependencia y existencia de programa institucional para el cuidado de la piel, entre otras. Finalmente, la sección III incluyó 12 preguntas relacionadas con datos sociodemográficos del paciente; su procedencia, la cual discriminó por provincias; diagnóstico principal relacionado con la dependencia física, así como información relacionada con las lesiones: ubicación, tipo de lesión, categoría, extensión, antigüedad, contexto donde se generó.

El protocolo de recolección de datos comprendió dos momentos para la aplicación de la encuesta, la cual se estructuró con las tres secciones descritas. En el primer momento se formulaban las preguntas relacionadas con la información institucional sección I y los datos sobre la medición del riesgo y la capacitación recibida para la clasificación de las lesiones asociadas a la dependencia de la sección II, teniendo como referente lo planteado por el GNEAUPP, los paneles NPUAP y EPUAP. En caso de que la persona que respondía la encuesta señalara no tener capacitación para clasificar las LPAD, se suministraba un tiempo adicional que correspondía al segundo momento del protocolo para que valorara la piel de los pacientes con la guía orientadora construida por las investigadoras a partir lineamientos del GNEAUPP y así facilitar el desarrollo de la sección III, donde se le indicaba que después de hacer una observación directa del paciente y comprobar la lesión con las imágenes de la guía orientadora podría relacionar y responder adecuadamente el tipo de lesión que presentaba cada una de las personas afectadas. $\mathrm{Si}$ quien respondía la encuesta refería tener capacitación para determinar el tipo de LPAD y su clasificación, procedía a responder la sección III de la encuesta con verificación visual de las lesiones. En ambos casos se finalizaba con la confirmación de algunos datos sociodemográficos del paciente, así como de la lesión en la historia clínica.

La muestra estuvo constituida por 409 pacientes hospitalizados, entre hombres y mujeres adultos ingresados en dos entidades de salud con servicio de hospitalización y residentes de dos instituciones geriátricas, de los cuales 24 presentaron LPAD en el momento de la medición. El procesamiento de los datos se realizó en el programa Excel ${ }^{\circ}$ y programa SPSS-v $23^{\circ}$ y comprendió análisis estadísticos univariados. 


\section{Consideraciones éticas}

Esta investigación surgió como subproyecto del macroproyecto: Abordaje integral de la persona, familia y comunidad, aprobado por el centro de investigación y extensión de la Facultad de Ciencias de la Salud de la Universidad Pedagógica y Tecnológica de Colombia. Contó con la aprobación de los comités de ética de cada institución participante. Por lo que respecta a la organización de los datos, se le otorgó un código consecutivo a cada caso, con el fin de garantizar el anonimato de la identidad de los pacientes y de las instituciones, según lo contemplado en la Ley 15/1999 de protección de datos de carácter personal. Igualmente, se contempló la resolución 8430 de 1993, que establece las normas científicas, técnicas y administrativas para la investigación, categorizándola como sin riesgo; igualmente, la normatividad de enfermería se tuvo en cuenta para el desarrollo del trabajo, principalmente en lo concerniente a los principios éticos de beneficencia, no maleficencia y autonomía.

\section{$\checkmark$ RESULTADOS}

Las instituciones de salud participantes corresponden a dos de tipo público y dos de tipo privado, con un índice ocupacional del $100 \%$ o más en el momento de la aplicación de la encuesta para el caso de tres instituciones, y una con 50\%. Las personas que diligenciaron la encuesta desempeñaban cargos asistenciales en su totalidad y para la recolección de algunos datos institucionales se contó con el apoyo del personal administrativo. Se encontró que una institución cuenta con programa de piel sana y clínica de heridas; las otras tres no poseen ningún programa institucionalizado relacionado con el cuidado de la piel. De igual manera, tres instituciones miden únicamente el riesgo de desarrollar LPP mediante la escala de Braden, y una no realiza medición de riesgo con ninguna escala. Cabe destacar que de las personas que respondieron la encuesta de prevalencia, tres eran profesionales de enfermería y una contaba con título de técnico en enfermería. En cuanto a la habilidad por parte del personal de salud para el reconocimiento y clasificación de las LPAD, únicamente un profesional de enfermería de los encuestados declaró que ostentaba la capacidad para identificar y clasificar las lesiones que presentaban sus pacientes, además de reconocer que había recibido capacitación previa al estudio para ese propósito, ya fuera por parte de la institución o por fuentes académicas autogestionadas. El resultado preocupa, dadas las implicaciones de las lesiones para las personas, sus familias, las instituciones y el sistema de salud, y al mismo tiempo genera importantes desafíos como reencaminar las estrategias de educación, el afrontamiento de la situación desde los líderes institucionales de calidad y seguridad del paciente, y el planteamiento de intervenciones pedagógicas dirigidas a los diferentes niveles de atención y hacia el equipo interdisciplinar de salud.

La información relacionada con las LPAD fue emitida por los enfermeros y las enfermeras encuestados después de realizar una observación directa de cada paciente afectado, seguido de la verificación en la historia clínica sistematizada o física de datos sociodemográficos, días de estancia hospitalaria del paciente y contexto en el que se generó la lesión(es), entre otros. La prevalencia de punto de LPAD fue del 5,86\% $(n=24)$. Respecto a la caracterización sociodemográfica de las personas afectadas, se encontró que, en cuanto al género, el 58,4\% eran mujeres y el 41,6\%, hombres; la edad media fue de 67,8 años, con un mínimo de 32 años y un máximo de 99 años, siendo los mayores de 50 años la edad más frecuente $(79,1 \%)$. En relación con la procedencia, el 53,8\% pertenecía al área urbana y el 46,2\% al área rural. El 56,6\% de los pacientes están afiliados al régimen subsidiado del sistema salud colombiano, mientras que el 30\% pertenecía al régimen contributivo y el 13,4\% a un régimen especial. Se encontró que las patologías que producían la dependencia física en las personas afectadas eran las fracturas de cadera $(25 \%)$, las enfermedades crónicas (20,8\%), el accidente cerebrovascular (12,5\%), la amputación de miembros inferiores $(8,3 \%)$, la artritis reumatoide $(8,3 \%)$, el traumatismo raquimedular $(8,3 \%)$, la polineuropatía $(8,3 \%)$, las quemaduras $(4,2)$ y la cirrosis $(4,2 \%)$.

Respecto a la caracterización de las heridas, se agruparon los datos según los criterios de clasificación dados por el EPUAP y el NPUAP. En este sentido, se detectaron 52 lesiones en 24 personas, lo que se traduce en un promedio de 2,1 lesiones por paciente afectado, siendo 7 el mayor número de lesiones presentadas en la misma persona. Del total de lesiones, $35(67,3 \%)$ fueron LPP en 7 pacientes, lo que representa una prevalencia del $1,71 \%$, mientras que de LESCAH se presentaron 7 lesiones $(13,4 \%)$ en 7 personas para una prevalencia del 1,71\%; en cuanto a desgarros cutáneos, se identificaron 4 lesiones en 4 sujetos, con una prevalencia del $0,97 \%$; en cuanto a las categorizadas por EPUAP como MARSI se encontraron 6 lesiones $(11,5 \%)$ en 6 personas para una prevalencia del 1,46\%. (tabla 1). En la agrupación de los datos se tuvo en cuenta que un paciente podía presentar varias LPAD en diferente estadio.

En relación con las lesiones por categorías establecidas, se encontró lo siguiente: para las LPP, el estadio que tuvo mayor frecuencia fue el grado I con un $25 \%$ del total de las lesiones; el estadio IV con el 11,5\%, seguido de lesiones de tejidos profundos y sin estadificar con un $7,7 \%$ y un $9,6 \%$, respectivamente. En cuanto las LESCAH que se categorizan según la integridad de la piel y área de afectación, en función del eritema se encontró: categoría IA con el 7,7\%, categoría IIA con afectación del 1,9\%, y para la categoría II B una afectación del 3,8\%. Para las lesiones MARSI, el porcentaje de afectación corresponde al 11,5\%. En el caso de las lesiones por desgarros cutáneos, el porcentaje corresponde al 7,7\% (tabla 2).

Tabla 1. Tipo de lesiones asociadas a la dependencia detectadas

\begin{tabular}{|l|c|c|c|}
\hline \multicolumn{1}{|c|}{ Tipo de lesión } & $N^{*}{ }^{0}$ & $(\%)$ & Prevalencia \\
\hline LPP & 35 & 67,30 & $1,71 \%$ \\
\hline LESCAH & 7 & 13,40 & $1,71 \%$ \\
\hline MARSI & 6 & 11,50 & $1,46 \%$ \\
\hline Desgarros cutáneos & 4 & 7,70 & $0,97 \%$ \\
\hline Total & 52 & 100,00 & $5,86 \%$ \\
\hline
\end{tabular}

LESCAH: lesiones por humedad; LPP: lesiones de piel por presión; MARSI: Medical Adhesive-Related Skin Injuries (lesiones de piel relacionadas con adhesivos médicos).

Tabla 2. Frecuencia de lesiones categorizadas

\begin{tabular}{|l|c|c|}
\hline \multicolumn{1}{|c|}{ Lesión categorizada } & $\mathbf{N}^{{ }^{0}}$ & $(\%)$ \\
\hline LPPI & 13 & 25 \\
\hline LPP II & 4 & 7,70 \\
\hline LPP III & 3 & 5,80 \\
\hline LPP VI & 6 & 11,50 \\
\hline No estadificable & 5 & 9,6 \\
\hline Lesión de tejidos profundos & 4 & 7,70 \\
\hline LESCAH I A & 4 & 7,7 \\
\hline LESCAH II A & 1 & 1,90 \\
\hline LESCAH III A & 2 & 3,80 \\
\hline MARSI & 6 & 11,50 \\
\hline Desgarros cutáneos & 4 & $7,70 \%$ \\
\hline Total & 52 & $100 \%$ \\
\hline
\end{tabular}

LESCAH: lesiones por humedad; LPP: lesiones de piel por presión; MARSI: Medical Adhesive-Related Skin Injuries (lesiones de piel relacionadas con adhesivos médicos). 
En relación con la región anatómica, las lesiones presentadas se distribuyeron de la siguiente manera: mayor porcentaje en miembros superiores con un 19,2\%; en igual proporción en región sacra con un 15,4\% y trocantérica con un $15,4 \%$; isquion, talón y escapular con el $21,2 \%$; en zona perineal, retroauricular y maléolos con el $11,5 \%$, y menor en pierna con el $9,6 \%$, y un 7,7\% para área occipital, frontal, abdominal y dorsovertebral. (tabla 3).

El origen de las lesiones fue catalogado como domiciliario en 30 $(57,7 \%)$, institucional en $20(38,5 \%)$ y generadas en otras instituciones 2 para un $3,8 \%$.

Tabla 3. Distribución de las lesiones de piel asociadas a la dependencia detectadas por localización anatómica

\begin{tabular}{|c|c|c|}
\hline Localización & $N^{0}$ & $(\%)$ \\
\hline Miembros superiores & 10 & 19,23 \\
\hline Sacro & 8 & 15,40 \\
\hline Trocánter & 8 & 15,40 \\
\hline Pierna & 5 & 9,61 \\
\hline Isquion & 4 & 7,69 \\
\hline Escápula & 3 & 5,80 \\
\hline Talón & 4 & 7,70 \\
\hline Perineal & 2 & 3,80 \\
\hline Retroauricular & 2 & 3,80 \\
\hline Maléolo & 2 & 3,80 \\
\hline Occipucio & 1 & 1,92 \\
\hline Frontal & 1 & 1,92 \\
\hline Abdominal & 1 & 1,92 \\
\hline Dorsovertebral & 1 & 1,92 \\
\hline Total & 52 & 100,00 \\
\hline
\end{tabular}

\section{У DISCUSIÓN}

Es elemental que desde los profesionales en salud se conciba esta problemática como un asunto de interés general, que desafortunadamente presenta tendencia al subregistro y a la desatención; de este modo, en esta investigación se abordó como un evento mínimamente estudiado en la región y se buscó determinar específicamente la prevalencia de punto de LPAD, con la intención de ir más allá de calcular el dato netamente epidemiológico y pasar a generar planes y programas de capacitación tanto al cuidador formal como informal, para lograr reducirlas o prevenirlas por sus altos costos humanos y económicos. Los resultados concuerdan con estudios similares realizados a nivel nacional, en especial en lo que respecta a los aspectos sociodemográficos y los tipos de lesión.

En la caracterización sociodemográfica, específicamente en la variable género, se encontró predominio de afectación por lesiones en mujeres; en cuanto a la edad, se identificó que las LPAD se presentan con mayor frecuencia en pacientes mayores de 50 años y que pertenecen al régimen subsidiado de salud, aspecto coincidente con estudios realizados en Colombia ${ }^{23}$. La prevalencia puntual encontrada en pacientes con heridas por dependencia en Tunja fue del 5,86\% $(n=24)$, mayor que la reportada en los estudios colombianos que la ubican entre el 2,2\% ${ }^{24}$ y el $5,2 \%{ }^{23}$. Igualmente, mayor que la encontrada en el estudio del hospital de Elche, donde únicamente se abordaron LPP con un $5,24 \%{ }^{27}$. Sin embargo, es menor que la reportada en el $5^{\circ}$ Estudio Nacional español, donde la prevalencia fue del $8,7 \%{ }^{16}$, y a la indicada en el estudio del Hospital de Sierrallana y Tres Mares, en Cantabria, con una prevalencia de punto para las LPAD del $17,1 \%(\mathrm{n}=27)^{28}$, así como a la obtenida en el Hospital Universitario de Burgos con 21,53\% ( $\mathrm{n}=99)$, en el que analizaron LPP y LESCAH en un periodo de tiempo ${ }^{17}$.

Según lo encontrado, las LPP ocupan el primer lugar de predominio de lesiones relacionadas con la dependencia física en relación con otros estudios que representan el 21,53\% en el Hospital de Burgos ${ }^{17}$ y el $43,1 \%$ en el estudio por departamentos de Colombia ${ }^{23}$. En relación con las lesiones MARSI, el presente estudio encontró un 11,5\% (prevalencia de $1,46 \%$ ) comparado con el 10,8\% del estudio colombiano ${ }^{23}$. Las LESCAH encontradas corresponden a una prevalencia del 1,71\%, menor frente a la encontrada en el Hospital de Burgos con un 5,69\% ${ }^{17}$. Para los desgarros cutáneos se halló un porcentaje del 7,7\% frente al $5,5 \%$ reportado por el estudio hecho para once departamentos colombianos, lo que revela una diferencia mínima entre los dos estudios para este aspecto y una aproximación fundamental, dado que ya se incluye este tipo de lesiones cutáneas, que antes no se tenían en cuenta.

Respecto al sitio anatómico, donde más se presentaron LPAD fue en los miembros superiores, específicamente en el antebrazo, con diez lesiones equivalente al 19,2\%, situación que difiere de varios estudios ${ }^{16,17,23,24}$, donde se revela el sacro y el talón como las zonas más afectadas. Realidad derivada de la condición de discapacidad y dependencia de las personas y asociada a la escasa enseñanza para los adecuados cambios de posición y lubricación de piel.

La literatura científica muestra que los estudios más frecuentes realizados respecto al tema son de LPP y no incluyen todas las LPAD; sin embargo, algunos evidencian una importante variación respecto a la estadificación. En cuanto al estadio I, en Tunja se reportaron frecuencias por encima del porcentaje nacional ${ }^{24}$, pero por debajo de lo que revelan los estudios españoles ${ }^{17,18}$.

Según el contexto en el cual se desarrollaron las lesiones, este estudio determinó que el $57,7 \%$ se generó en un contexto domiciliario, resultado que difiere de los reportados en México $\mathrm{DF}^{29}$, en donde la mayoría de las lesiones se formaron dentro de la misma institución, con un $81,4 \%$, así como a lo determinado en el estudio realizado en el Hospital de Burgos, donde el $88,8 \%$ se generó en el entorno hospitalario. Eso indica la necesidad de trabajar la educación en salud para egreso hospitalario y cuidado en casa.

Los resultados del presente estudio son novedosos y constituyen un referente fundamental de la problemática, aunque podría considerarse una prevalencia baja que puede estar influenciada por ciertas limitaciones, como que se constituyó una muestra pequeña, además de la probabilidad de restricciones en el reporte total de lesiones existentes en todos los pacientes, por motivos relacionados con la habilidad del encuestado, así como prevenciones por posibles sanciones a nivel institucional.

\section{$\searrow$ CONCLUSIONES}

La prevalencia de LPAD reportada en esta investigación es menor que la revelada en estudios internacionales y corresponde a la publicada en estudios nacionales previos, donde no se incluyeron datos locales. Las mujeres y las personas mayores de 50 años son quienes más resultan afectadas por esta problemática, situación que sugiere un llamado a la adopción de prácticas de cuidado seguras que beneficien a los adultos mayores en la cuidad de Tunja.

De igual manera, si se tiene en cuenta que es un estudio que revela la problemática en un momento puntual, constituye un indicador impor- 
tante, básico para la formulación de planes de prevención; así mismo, demuestra que las LPP siguen siendo las que generan mayor afectación a los pacientes con dependencia.

Es fundamental generar estrategias educativas basadas en los lineamientos GNEAUPP, NPUAP y EPUAP que involucren al equipo de salud interdisciplinario, a los profesionales en formación, especialmente a los de áreas de la salud, a pacientes y cuidadores, para fortalecer la medición del riesgo, la prevención, clasificación y manejo efectivo.
Se resalta que no existen muchos estudios en la literatura que incluyan la prevalencia de todas las lesiones asociadas a la dependencia propuestas por el modelo español de García Fernández; por tal motivo, este estudio contribuye a la investigación en el área, pues evidencia una aproximación a los adelantos científicos generados por estudiosos de la disciplina enfermera

\section{Conflicto de intereses}

Las autoras declaran no tener ningún conflicto de intereses.

\section{У BIBLIOGRAFÍA}

1. García-Fernández FP, Agreda JJ, Verdu J, Pancobo-Hidalgo PL. A new Theorical Model for the Development of Pressure Ulcers and Other Dependence-Related Lesions. Journal Nursing Scholarhip. 2013:46(1):28-38. PubMed PMID-24118691.

2. García-Fernández FP, Soldevilla Agreda JJ, Pancobo-Hidalgo PL, Verdú J, López Casanova P, Rodríguez Palma M. Clasificación-categorización de las lesiones relacionadas con la dependencia. Serie de documentos técnicos GNEAUPP n II. GNEAUPP [Internet]. 2014 [consultado 20 de diciembre de 2018]. Disponible en https://gneaupp.info/wp-content/uploads/2014/12/clasificacion-categorizacion-de-las-lesiones-relacionadas-con-la-dependencia-segunda-edicion.pdf

3. Zulkowski K. Understanding Moisture-Associated Skin Damage, Medical Adhesive-Related Skin Injuries, and Skin Tears. Adv Skin Care [internet]. 2017 [consultado 21 de diciembre de 2018];30(8):372-81. https://doi:10.1097/01. ASW.0000521048.64537.6e

4. Rumbo JM, Aranton L, López de los Reyes R, Vives E, Palomar $F$, Cortizas JS. Valoración y manejo integral de las lesiones cutáneas asociadas a la humedad (LESCAH). Enferm Dermatol [internet]. 2015 [consultado 29 de septiembre de 2018]:9(25):1730. Disponible en: https://dialnet.unirioja.es/servlet/articulo?codigo $=5423375$

5. Benítez-Agudelo Juan C, Barceló-Martínez Ernesto A Gelves-0spina M. Características psicológicas de los pacientes con larga estancia hospitalaria y propuesta de protocolo para su manejo clínico. Cir. plást. Iberolatinoam [Internet]. 2016 [consultado 20 agosto 2019]:42(4):391-8. Disponible en: http://dx.doi. org/10.4321/S0376-78922016000400012

6. Soldevilla Agreda JJ. Una epidemia viva del siglo XXI. Úlceras por presión <<como problema〉>. El farmacéutico [internet]. 2010 [consultado 15 de octubre de 2018];437:39-48. Disponible en: https://gneaupp.info/salud-publica-las-ulceras-por-presioncomo-problema-una-epidemia-viva-y-silenciosa-en-el-siglo-xxi/

7. Tamayo-Rendón CM, Palacio-Grisales C, Osorio-Pabón CM, Román-Giraldo JE, Rodríguez-Castrillón LC. Comportamiento de las úlceras por presión en los servicios de unidad de cuidados intensivos y unidad cuidados especiales en una clínica de alta complejidad de la ciudad de Medellín durante el primer semestre de 2014. CES Salud Pública [internet]. 2015 [consultado 4 noviembre 2018]:6(1):27-34. Disponible en: http://revistas ces. edu.co/index.php/ces_salud_publica/article/view/3116/2403

8. Stegensek Mejía EM, Jiménez Mendoza A, Romero Gálvez LE, Aparicio Aguilar A. Úlceras por presión en diversos servicios de un hospital de segundo nivel de atención. Enfermería Universitaria [internet]. 2015 [citado 8 de junio 2019];12(4):173-81. http://dx.doi.org/10.1016/j.reu.2015.08.004

9. Cabello Granado PA, Arévalo-Velasco JM. Estimación del coste sanitario de las úlceras por presión en pacientes lesionados medulares. Rev. Gerenc. Polít. Salud. [internet]. 2016 [citado el 18 febrero de 2019];15(30):60-7. http://dx.doi. org/10.11144/ Javeriana.rgyps15-30.ecsu
10. Prevención úlceras por presión. Paquetes instruccionales. Guía técnica "Buenas prácticas para la seguridad del paciente en la atención en salud" versión 2.0. Ministerio de Salud. Disponible en: https://www.minsalud.gov.co/sites/rid/Lists/BibliotecaDigital/RIDE/DE/CA/prevenir-ulceras-por-presion.pdf

11. Borsting TE, Tvedt CR, Skogestad IJ, Granheim TI, Gay CL, Lerdal A. Prevalence of pressure ulcer and associated risk factors in middle- and older-aged medical inpatients in Norway. J Clin Nurs [internet]. 2018 [citado 6 de enero de 2019];27(3-4):53543. doi: 10.1111/jocn.14088. https://www.ncbi.nlm.nih.gov/ pubmed/28960642

12. Kayser SA, Van Gilder CA, Ayello EA, Lachenbruch C. Prevalence and Analysis of Medical Device-Related Pressure Injuries: Results from the International Pressure Ulcer Prevalence Survey. Adv Skin Wound Care [Internet]. 2018 [citado 3 de mayo de 2019];31(6):276-85. doi: 10.1097/01.ASW.0000532475.11971. aa

13. Kayser SA, Phipps LA, Van Gilder CA, Lachenbruch C. Examining Prevalence and Risk Factors of Incontinence-Associated Dermatitis Using the International Pressure Ulcer Prevalence Survey. J Wound Ostomy Continence Nurs [internet]. 2019 [citado 1 de agosto de 2019];46(4):285-90. doi: 10.1097/ WON.0000000000000548

14. Latimer S, Chaboyer W, Lukman Thalib L, McInnes E, Buckna$\| T$, Gillespie BM. Pressure injury prevalence and predictors among older adults in the first 36 hours of hospitalization. J Clin Nurs [internet]. 2019 [citado 28 Julio de 2019]. doi: 10.1111/ jocn. 14967

15. Chaboyer W, Bucknall T, Webster J, McInnes E, Gillespie BM, Banks $M$, et al. The effect of a patient centred care bundle intervention on pressure ulcer incidence (INTACT): A cluster randomised trial. Int J Nurs Stud [internet] 2016 [citado 20 octubre 2018];23(64):63-71. doi: 10.1016

16. Pancorbo-Hidalgo PL, García-Fernández FP, Pérez-López C, Soldevilla Agreda JJ. Prevalencia de lesiones por presión y otras lesiones cutáneas relacionadas con la dependencia en población adulta en hospitales españoles: resultados del $5^{\circ}$ Estudio Nacional de 2017. Gerokomos. 2019:30(2):76-86.

17. Real López L, Diez Estébanez ME, Serrano Hernantes M, De la Iglesia García E, Blasco Romero I, Capa Santamaría S, et al. Prevalencia de úlceras por presión y lesiones cutáneas asociadas a la humedad en el Hospital Universitario de Burgos. Gerokomos [internet]. 2017 [citado 23 marzo 2019];28(2):1038. Disponible en: http://gerokomos. com/wp-content/uploads/2017/07/28-2-2017-103.pdf

18. Labiano-Turrillas J Larrea-Leoz B Vázquez-Calatayud M Juandeaburre-Pedroarena B, Irruarizaga-Sagredo A, Morillo-Cabezas E. Estudio de prevalencia de úlceras por presión en la Clínica Universidad de Navarra. Gerokomos 2013;24(4):184-8. http:// dx.doi.org/10.4321/S1134-928X2013000400007

19. Martínez Valle I. Prevalencia de úlceras por presión en la población mayor de 75 años de Fuenterrobles. Enferm Dermatol [internet]. 2017 [citado 18 mayo 2019];11(31):26-35. Disponible en: https://dialnet.unirioja.es/servlet/articulo?codigo=6324067
20. Organización Panamericana de la Salud, Organización Mundial de la Salud. Úlceras por presión de pacientes en estado no crítico en un hospital de 3 nivel. Una encuesta transversal. México: OPS OMS: 2016. Boletín BOLETIN CONAMED - OPS: 9. Disponible en: http://www.conamed.gob.mx/gobmx/boletin/ pdf/boleting/ulceras_presion.pdf

21. Vieira CPB, Araújo TME. Prevalence and factors associated with chronic wounds in older adults in primary care. Rev Esc Enferm [Internet]. 2018 [citado 8 de junio 2019];52:e03415. doi: 10.1590/S1980-220X2017051303415

22. Sánchez-Cruz $L Y$, Martínez-Villarreal AA, Lozano-Platonoff $A$ Cárdenas-Sánchez A, Contreras-Ruiz II J. Epidemiología de las úlceras cutáneas en Latinoamérica. Med Cutan Iber Lat Am [Internet]. 2016 [citado en 22 mavo 2019]:44(3):183-97. Disponible en: www.medigraphic.com/medicinacutanea

23. González Consuegra RV, López Zuluaga WJ, Roa Lizcano KT Epidemiología de lesiones de piel relacionadas con el cuidado: estudio de la prevalencia en Colombia. Revista de Enfermagem [internet]. 2017 [citado el 12 noviembre 2018];4(15):65-72. https://doi.org/10.212707/RIV17038

24. González-Consuegra RV, Cardona-Mazo DM, Murcia-Trujillo PA, Matiz-Vera GD. Prevalencia de úlceras por presión en Colombia: informe preliminar. Rev. Fac. Med [internet]. 2014 [citado 14 de noviembre de 2018):62(3):369-77. doi: http://dx.doi. org/10.15446/revfacmed.v62n3.43004

25. González-Consuegra RV, Roa-Lizcano KT, López-ZuluagaWJ. Estudio de prevalencia de lesiones por presión en un Hospita Universitario, Bogotá-Colombia. Rev. cienc. Cuidad [internet] 2018 [citado 12 de febrero de 2019];15(2):92-102. doi: http:// dx.doi.org/10.22463/17949831.1404

26. Torra-Bou JE, Verdú-Soriano J, Sarabia-Lavin R, Paras-Bravo P, Soldevilla-Ágreda JJ, García-Fernández FP. Las úlceras po presión como problema de seguridad del paciente. Gerokomos [Internet]. 2016 [citado 1 de julio de 2019];27(4):161-7. Disponible en: http://scielo.isciii.es/scielo.php?script=sci_arttext\&pi$d=\$ 1134-928 X 2016000400007 \&$ lng=es

27. Talens Belén F. Formación y prevención en úlceras por presión: prevalencia en el Hospital General de Elche. Gerokomos [internet] 2015 [citado 22 de agosto de 2019];27(1):33-7. Disponible en: http:// gerokomos.com/wp-content/uploads/2016/07/27-1-2016-033.pdf

28. Olavarria Beivide E, Gómez Muñoz I, Cacicedo González R, Revuelta Arroyo R, Parás Bravo P, Herrero Montes M. Prevalencia de lesiones relacionadas con la dependencia en pacientes hospitalizados. En: XIX Encuentro Internacional de Investigación y $19^{\text {th }}$ Internacional Nursing Research Conference. Cuenca, España: Instituto de Salud Carlos III; 2015. 285-592/ ponencia.

29. Barrera Arenas JE, Pedraza Castañeda MC, Pérez Jiménez G, Hernández Jiménez P. Reves Rodríguez JA, Padilla Zárate MP. Prevalencia de úlceras por presión en un hospital de tercer nivel, en México DF. Gerokomos [Internet]. 2016 [citado 22 de junio de 2019];27(4):176-81. Disponible en: http://gerokomos.com/ wp-content/uploads/2017/01/27-4-2016-176.pdf 